\title{
Development of soft tactile sensor for prostatic palpation diagnosis: sensor structure design and analysis
}

\author{
Zhongwei Jiang $^{\mathrm{a}, *}$, Seiji Chonan ${ }^{\mathrm{b}}$, \\ Yoshikatu Tanahashi ${ }^{\mathrm{c}}$, Mami Tanaka ${ }^{\mathrm{b}}$ and \\ Takuji Kato ${ }^{\mathrm{b}}$ \\ ${ }^{a}$ Department of Mechanical Engineering, Yamaguchi \\ University, Ube, 755-8611, Japan \\ ${ }^{\mathrm{b}}$ Department of Mechatronics and Precision \\ Engineering, Tohoku University, Aoba-yama 04, \\ Sendai, 980-8579, Japan \\ ' Tohoku Kousai Hospital, Aoba-ku Kokubun-Cho \\ 2-3-11, Sendai, Japan
}

Received 13 September 1999

This paper is concerned with the development of a palpation probe for assessment of prostatic cancer and hypertrophy. The sensor is constructed by layering two polivinylidene fluoride (PVDF) films into soft material layers such as vulcanized rubber and sponge rubber sheets. Since the contact condition between the sensor and soft objects influences the sensor output significantly, the improvement of its sensitivity is investigated by design of an attachment covered over the sensor surface. In this report, the theoretical model of the sensor and the design of the attachment are presented and the attachment is validated for soft substances due to experimental investigation. Finally, a compact probe developed for clinical testing is presented and the data obtained from the patients are treated by the wavelet transform for de-noising. Some representative clinical data show that the sensor could be a useful tool for group medical checkup on prostatic cancer and hypertrophy.

\section{Introduction}

Prostatic cancer and hypertrophy are recently diagnosed by ultrasonic endoscope together with the help of rectal palpation by doctor's forefinger. Prostatic hy-

\footnotetext{
*Corresponding author. Tel. and Fax: +81 83635 9913; E-mail: jiang@po.cc.yamaguchi-u.ac.jp.
}

pertrophy occurs as a soft nontumorous enlargement while prostatic carcinoma presents as a hard, asymmetric uneven swell. In the examination of prostate gland, the doctor usually first uses the ultrasonic endoscope checking the shape of prostate gland and detecting alien substances in it by the tomography. After abnormal change was recognized, the rectal palpation is made by doctor's forefinger for examining its physical properties, such as the softness and the swell of the gland, to assess whether the prostatic hypertrophy or cancer based on his experience. If there is any possibility to be prostate cancer the tissue of the gland is taken for a close examination. Since the tactile perception of the finger is ambiguous and it depends on each individual person, the rate of correct diagnosis is, therefore, greatly affected by the examiner's experience or his fitness. To develop the objective sensing systems for noninvasive assessment of the human organs mechanical behavior in vivo, numerous attempts were made during the two last decades. The major devices were constructed which operate either by inducing a deformation and recording the resisting force or by putting a load and assessing the resulting deformation. Rigal and Leveque [9] studied a measurement of skin elasticity by using a torsional technique. A small disc rotated by a torque motor was glued to the skin and the skin angular deformation in time upon application of a constant torque was measured to assess the skin mechanical properties. Anther method for measuring the skin mechanical properties was by the suction force exerted over a defined area of the skin [2]. In this case, the skin elevation in the function of the suction time or the suction force was measured to calculate various parameters of skin mechanical properties. In striking contrast to the suction method, a balloon-type sensor [12] was constructed with several conductive rubber strain gauges and it was inserted into the large intestine and swollen out against the wall of the intestine. The large intestine cancer was supposed to be quantified by cal- 
culating the output from the strain gauges. This sensor is assumed to be available for measuring a lump on the wall of duct organs.

In mechanical or materials engineering, the determination of stress (applied force) versus strain (resulting deformation) was used to describe the characteristics of any structural substance. As the extension of this principle, an automatic palpation system was developed for detecting the breast cancer instead of the hand [4]. It was constructed by four sensory rods, which were operated sinking in mamma by their own weight. The sinking distances in the function of time were measured to evaluate the tumor's hardness in the mamma. Further, a small vibrometer constructed with a piezoelectric disk and a pickup was used to assess the contact compliance of a soft body [6-8]. It was found that the contact compliances of soft materials could be described by the nondimensionalized frequency factor and mass factor, which have few influence on the weight of the vibrometer. However, this piezoelectric vibrometer was supposed to be put freely on the surface of the object, so it is inevitable that the pressure between the vibrometer and objects would change the output of the sensor if one was handling the vibrometer and pushed it against the object. Furthermore, for high frequency technique application on assessment of the properties of soft substances, Tanaka et al. [11] were studied a ultrasonic vibrator for noninvasively measuring the muscle stiffness. The vibrator was made of a piezoelectric device and the contact impedance of the vibrator and the measuring substance was recorded by impedance analyzer as the function of frequency in the range from 10 to $100 \mathrm{kHz}$, and then converted to the parameters describing the stiffness of the substance.

As for hardness measurement of prostate, Kosawada, et al. [5] tried to evaluate the dynamic characteristics of prostate tissue using a miniature cantilever beam. In this study comes that the pathological changes in prostate would give an alteration in its viscoelasticity; hence the property of the prostate tissue could be characterized by measuring the damped vibration of the miniature cantilever at tip of which is touched by the prostatic tissue.

So far, there are numerous methods for assessment of the mechanical properties of soft substances, and a lot of devices were developed for measurement of the hardness, especially for measurement of the skin elastic properties. However, the development is still beyond the demands for noninvasive assessment of the properties of human organs and for quantification of the palpation experience of doctors. Also according to the authors' knowledge no literature has been presented on the development of a palpation probe for measuring the softness of prostate gland. The attempt of this study aims to develop a simple sensor system that has sense of the human hands and could be the alternate to the doctors' forefinger palpation.

According to our study on polyvinylidene fluoride (PVDF) film it is found the output current from PVDF is proportional to the rate of the strain induced in the film. Experimental analysis has shown that the output voltage of the polymer film takes the form of a brief potential wave at the onset of the pressure pulse and has no response during the stationary plateau of the applied pressure, which is similar to the brief wave at the Pacinian corpuscle, a sensory receptor in the dermis [10]. Recently, the authors have assembled a tactile sensor made of PVDF films laminated between soft rubber sheets [3]. The output signals from the sensor were measured when the sensor was mounted on a displacement machinery pressing sinusoidally with a constant amplitude against various kinds of soft materials. The experimental results have shown the feasibility that the sensor could be used to distinguish the different hardnesses of the materials. However, the sensor output was greatly influenced by the contact condition between the sensor and measuring substances as well as the motion of the sensor if the sensor was operated by the hand. Further, how the sensor structure affecting its output was not presented in detail in the previous work.

In view of the above points, this paper is concerned with theoretical and experimental studies on sensor structure that is supposed to fit on the doctor's finger in rectal palpation of prostate gland. First, the fundamental characteristics of the sensor with a flat surface are examined and a theoretical analysis is conducted and discussed in detail in relating to the design of the sensor structure. Here, a new definition on softness is given based on the touching sensation of the finger, because the sensor system proposed is considered to be a substitution for the doctor's palpation. Then, several surface attachments overlaid on the sensor are designed based on the theoretical analysis, and the ratio of the two PVDF outputs is examined for its sensitivity to different material softnesses. Finally, a compact cylindrical sensor, which is fitted onto the doctor's forefinger, is developed and the result of preliminary clinical testing on patients is presented. Some representative clinical data on the hardened and normal prostate glands are collected and treated by wavelet transform analysis. The results verified that the present sensor seems useful for group medical checkup on prostate glands. 
Table 1

Young's moduli of sample materials

\begin{tabular}{ll}
\hline Specimen & Young's modulus \\
\hline Sponge rubber & $4.7 \times 10^{5} \mathrm{~N} / \mathrm{m}^{2}$ \\
Plastic foam & $1.5 \times 10^{6} \mathrm{~N} / \mathrm{m}^{2}$ \\
Vulcanized rubber & $4.9 \times 10^{6} \mathrm{~N} / \mathrm{m}^{2}$ \\
\hline
\end{tabular}

\section{Simple two-layer PVDF sensor}

First, a simple rectangular sensor as shown in Fig. 1(a) was fabricated to investigate the fundamental characteristics of the sensor output. The sensor has two PVDF films, denoted by PVDF1 and PVDF2, sandwiched by three rectangular sponge rubber sheets of $15 \mathrm{~mm} \times 20 \mathrm{~mm}$.

The experimental setup for the fundamental test of the sensor is shown in Fig. 2. Crank machinery was used so that a prescribed displacement could be obtained constantly by different sized cranks. The sensor was attached onto the translation stage and pressed sinusoidally with the prescribed displacement against the testing substance that is fixed in space. The output voltage signals from the PVDF films were measured by a digital storage oscilloscope and then sent to a personal computer for data processing. A strain gauge sensor as shown in the figure was also installed to monitor the applied force. Three materials were picked as the testing substances: sponge rubber, plastic foam and vulcanized rubber, with several thicknesses. Their Young's moduli are listed in Table 1 for reference.

Since the output of PVDF film is easily overwhelmed by the noise the temporal average of the absolute output signal was used as an index $I_{k}$ for evaluation of the softness of the substances [3], i.e.,

$$
\begin{aligned}
& I_{k}=\frac{1}{T} \sum_{n=1}^{N}\left|V_{k}(n)\right|, \\
& N=500, T=500 \mathrm{msec}, k=\left\{\begin{array}{l}
1 \text { for PVDF1, } \\
2 \text { for PVDF2. }
\end{array}\right.
\end{aligned}
$$

Here, $V_{k}$ is the output voltage, $n$ the number of data and $\Delta t$ the sampling time.

Figure 3 shows the outputs when the sensor is moved against a flat plastic foam with different driving frequencies and displacements. It was found that the outputs of $\operatorname{PVDF} 1\left(I_{1}\right)$ and $\operatorname{PVDF} 2\left(I_{2}\right)$ are almost the same and they increase proportionally with frequency and displacement. Figure 4 shows another example that the sensor output (for example, $I_{1}$ ) to the sponge rubbers with $20 \mathrm{~mm}$ thickness (Fig. 4b) is lower than the one of the same material but different thickness of $10 \mathrm{~mm}$ (Fig. 4a), which indicates the thickness of the mea- suring substances should be considered as a parameter involved in the sensor output.

Figure 5 illustrates the output of PVDF2 $\left(I_{2}\right)$ versus the output of PVDF1 $\left(I_{1}\right)$ when the flat sensor touches a plane surface substance as shown in Fig. 1b. Figure $5 \mathrm{a}$ is the results when the pushing stroke of sensor is $1.0 \mathrm{~mm}$, and Fig. 5b the corresponding results of the stroke $2.0 \mathrm{~mm}$. The symbols $\boldsymbol{\square}$ and $\downarrow$ indicate the index for the plastic foams of thickness of $10 \mathrm{~mm}, 20 \mathrm{~mm}$ and $30 \mathrm{~mm}$, and the symbols $\mathrm{O}, \square$ and $\diamond$ are the results for the sponge rubbers of thickness $10 \mathrm{~mm}, 20 \mathrm{~mm}$ and $25 \mathrm{~mm}$, respectively. The output $I_{1}$ and $I_{2}$ fall on the line $I_{2} / I_{1}$ so it is difficult to distinguish between the plastic foam and the sponge rubber. It means that the deformation in the sensor is homogeneous when the measuring objects have a plane surface.

In contrast with the plane surface object, a triangular object (see Fig. 1c) was tested. In this case, bending deformation is induced in the polymer films in addition to the homogeneous inplane deformation due to Poisson's effect. Figure 6 illustrates the variations of $I_{1}$ and $I_{2}$ with an increase of the oscillation frequency. The outputs of the surface film (PVDF1) are similar for the plastic foam $(\bigcirc)$ and the sponge rubber $(O)$, but the output of PVDF2 for the plastic foam is greater than the one for the soft sponge rubber. Figure $7 \mathrm{a}$ is a plot of $I_{2}$ versus $I_{1}$ for a pushing stroke of $2 \mathrm{~mm}$. It is clear that the difference between the plastic form and the sponge rubber can be evaluated by the ratio of $I_{2}$ to $I_{1}$. The experiment was also carried out for pushing strokes of $1 \mathrm{~mm}$ and $2.4 \mathrm{~mm}$ (Fig. 7b). The same results were obtained for several substances and it shows that the ratio $I_{2} / I_{1}$ could be the index for evaluation of substance softnesses regardless of the pushing stroke, strictly speaking, in a certain range. The above results suggest that the plane sensor can distinguish the softness of an object if it has a protuberance or a tumor on the surface.

\section{Analysis and design of sensor structure}

In order to construct an optimal designed sensor structure, an analysis model was considered as shown in Fig. 8. In the analysis, the strain in $i$ th element is defined by the applied force $F$ and the flexural rigidity $E_{i} A_{i}$, i.e.,

$$
\varepsilon_{i}=\frac{u_{i}-u_{i+1}}{l_{i}}=\frac{F}{E_{i} A_{i}}
$$




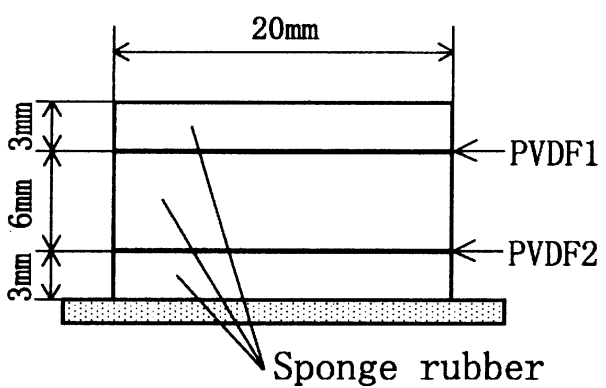

(a) plane sensor

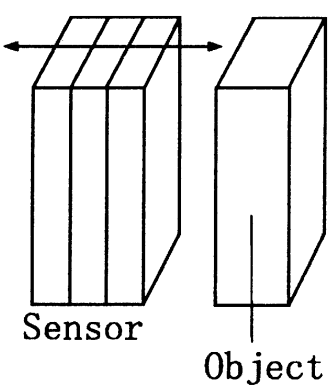

(b) flat object

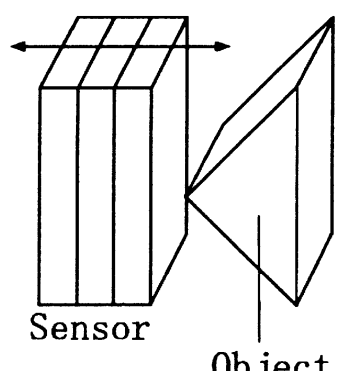

Object

Fig. 1. Rectangular sensor with plane surface and objects.

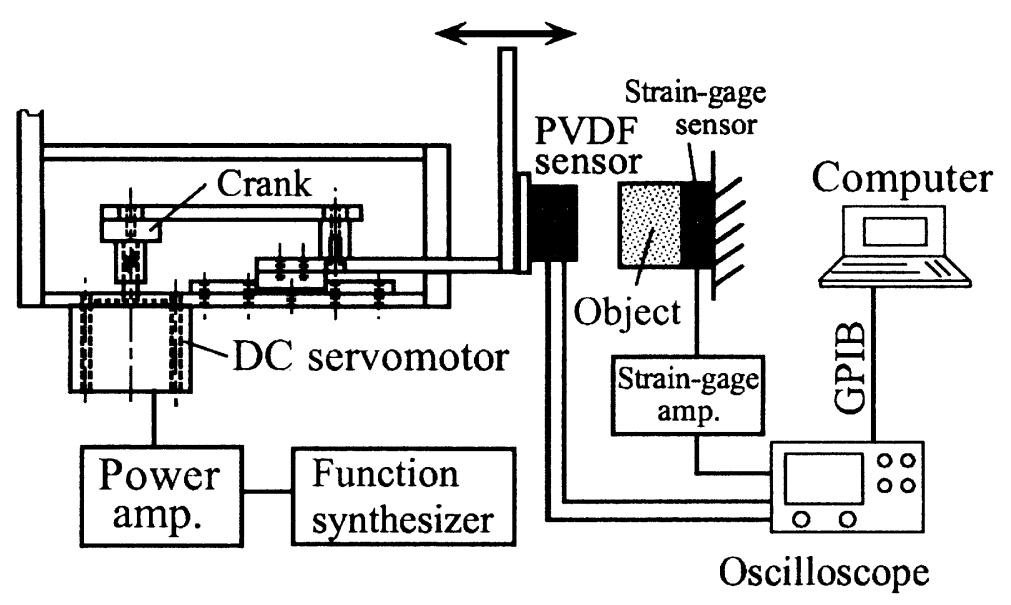

Fig. 2. Experimental setup for measuring fundamental characteristics of sensor.

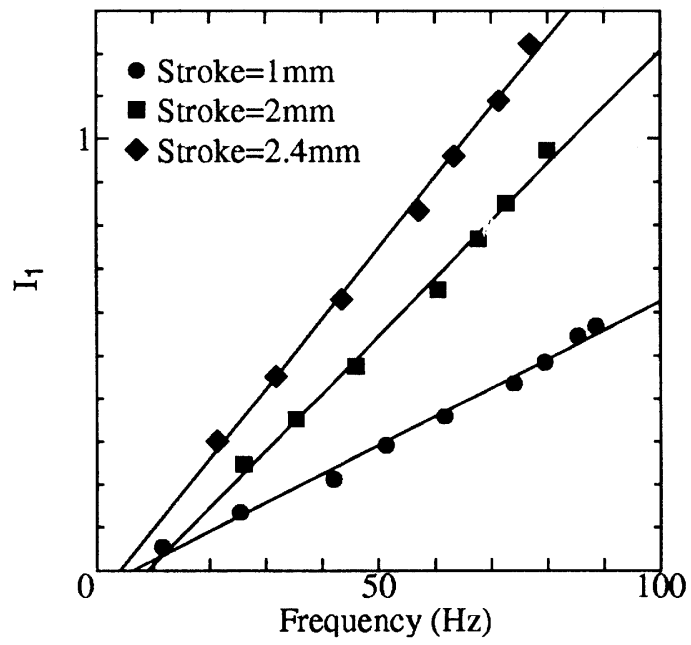

(a) output of PVDF1

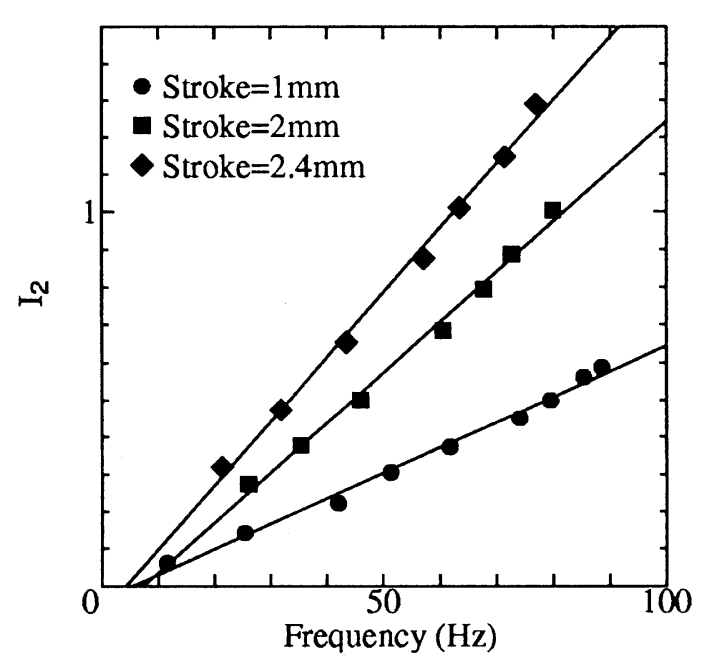

(b) output of PVDF2

Fig. 3. Frequency responses of PVDF1 $\left(I_{1}\right)$ and PVDF2 $\left(I_{2}\right)$ on a plane plastic foam block with various strokes. 


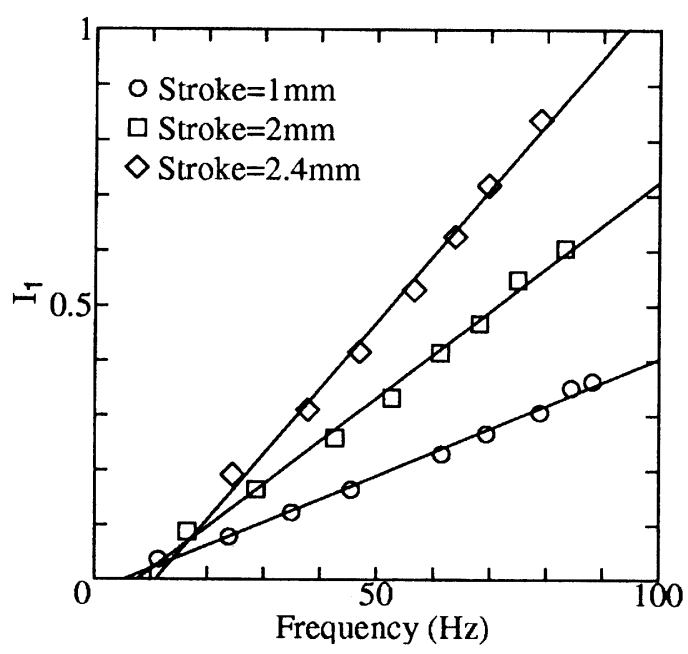

(a) $10 \mathrm{~mm}$ thickness

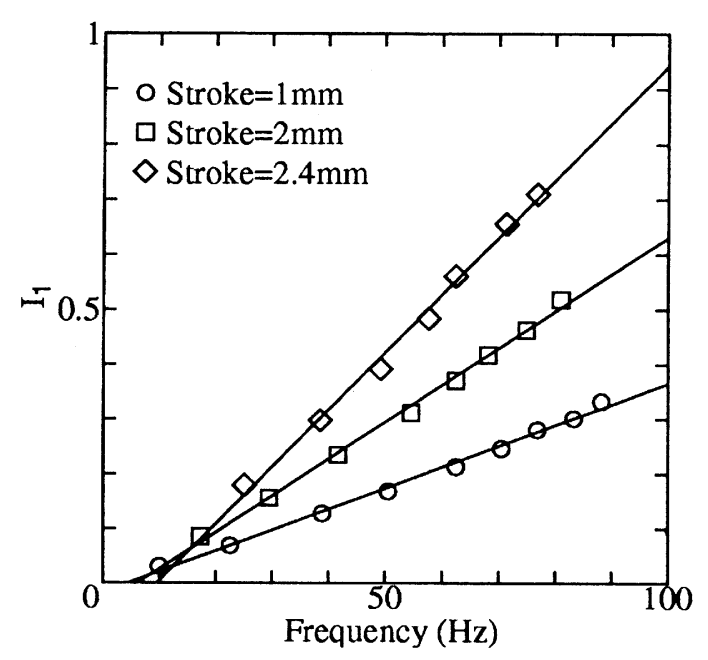

(b) $20 \mathrm{~mm}$ thickness

Fig. 4. Frequency response of PVDF1 $\left(I_{1}\right)$ on the same plane sponge rubber blocks but different in thickness.

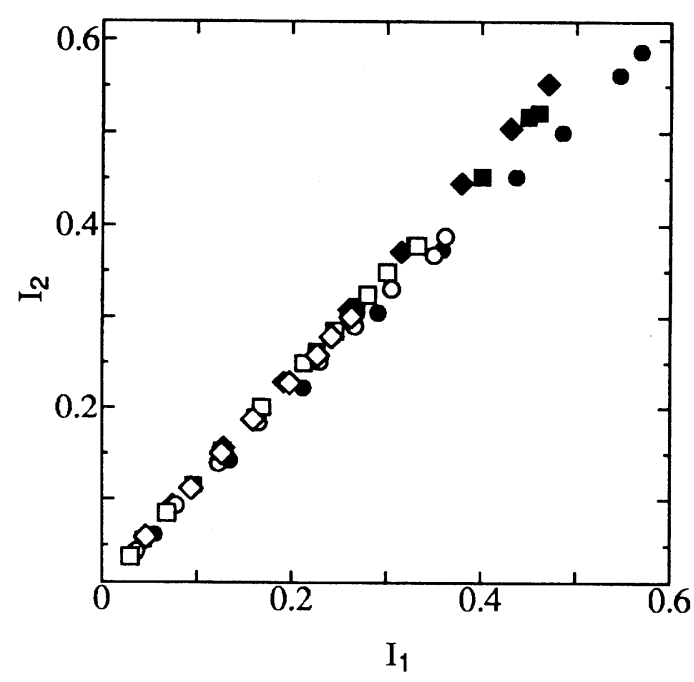

(a) stroke $=1.0 \mathrm{~mm}$

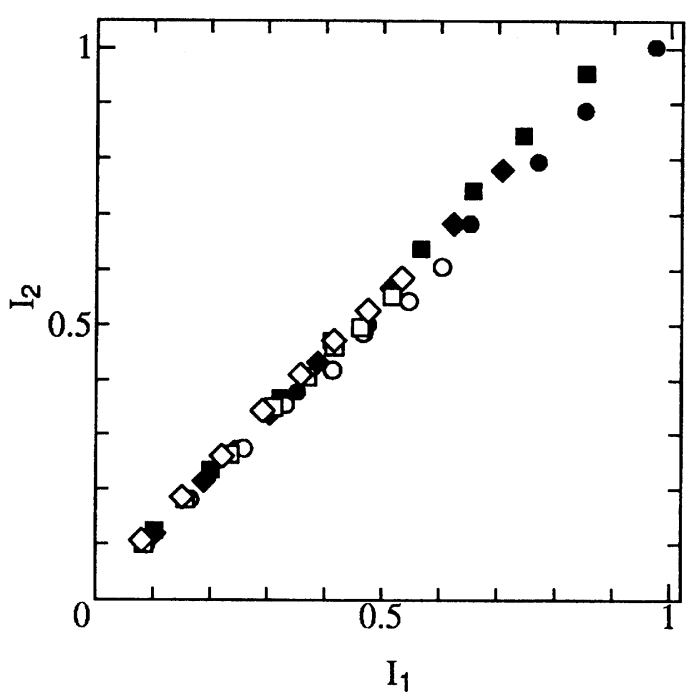

(b) stroke $=2.0 \mathrm{~mm}$

Fig. 5. Output of PVDF1 $\left(I_{1}\right)$ versus that of PVDF2 $\left(I_{2}\right)$ on plastic foams of $10 \mathrm{~mm}$ rubbers of $10 \mathrm{~mm}(\bigcirc), 20 \mathrm{~mm}(\square)$ and $25 \mathrm{~mm}(\diamond)$ thickness.

then the displacements $u_{i}$ and the deflections $\delta_{i}$ of each elements are given by

$$
\begin{aligned}
u_{i} & =u_{1} \sum_{k=i}^{4}\left(l_{k} / E_{k}\right) / \sum_{k=1}^{4}\left(l_{k} / E_{k}\right) \\
& =u_{1} \sum_{k=i}^{4} S_{k}^{F} / \sum_{k=1}^{4} S_{k}^{F}
\end{aligned}
$$

$$
\begin{aligned}
\delta_{i} & =\varepsilon_{i} l_{i}=u_{1}\left(l_{i} / E_{i}\right) / \sum_{k=1}^{4}\left(l_{k} / E_{k}\right) \\
& =u_{1} S_{i}^{F} / \sum_{k=1}^{4} S_{k}^{F}
\end{aligned}
$$

Here, considering a human's touch sense, we defined the softness $S^{F}$ of an object by the ratio of the thickness $l$ to the Young's Modulus $E$, just like for the same 


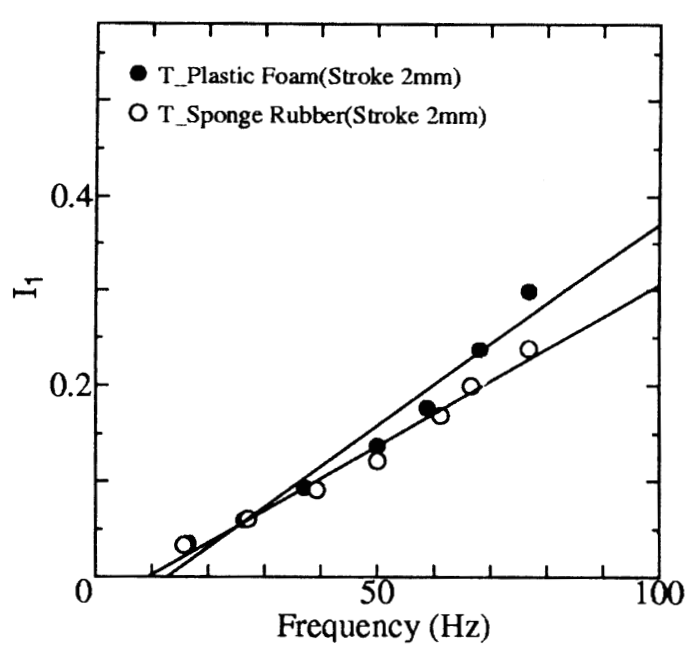

(a) output of PVDF1

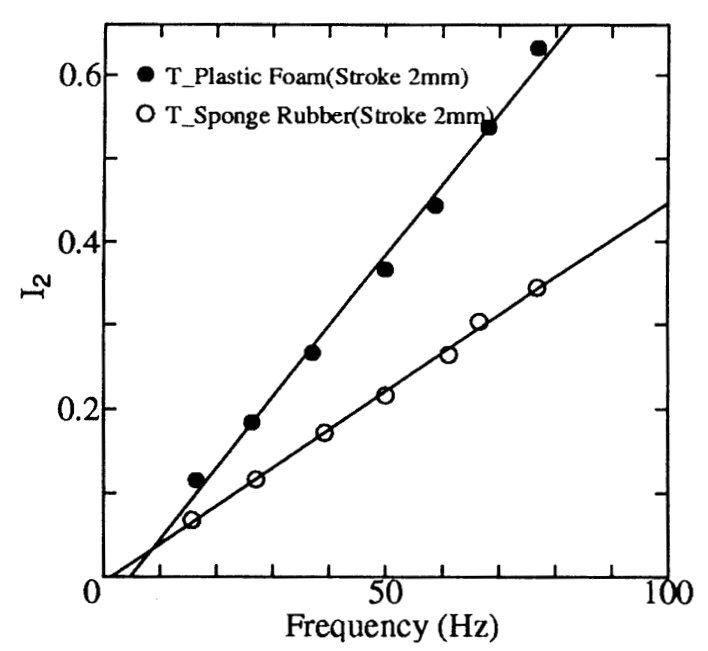

(b) output of PVDF2

Fig. 6. Frequency responses of PVDF1 $\left(I_{1}\right)$ and PVDF2 $\left(I_{2}\right)$ on triangular objects of plastic foam and sponge rubber.

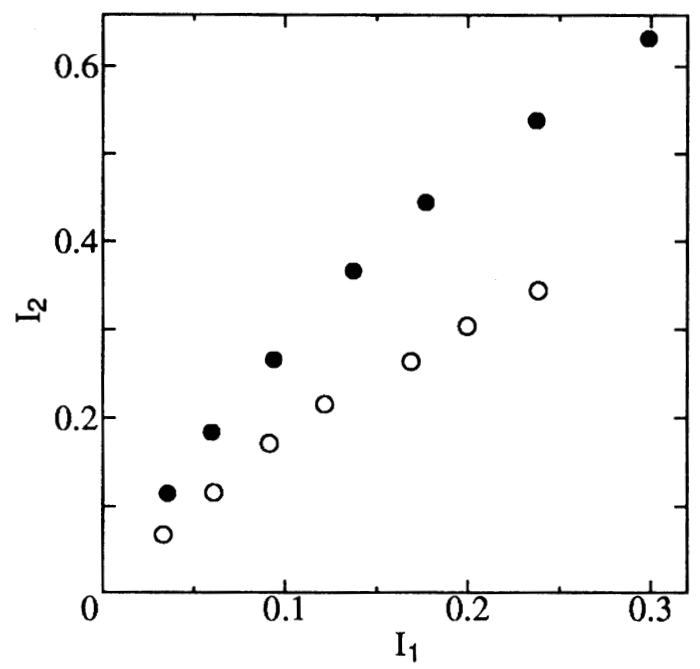

(a) redisplay of Fig. 6 by $I_{1}$ and $I_{2}$

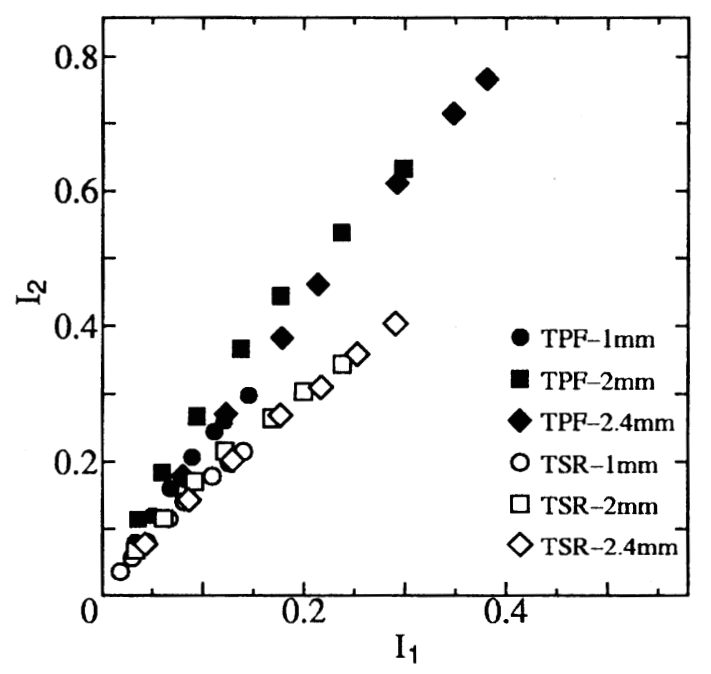

(b) with various strokes $(1,2,2.4 \mathrm{~mm})$

Fig. 7. Output of PVDF1 $\left(I_{1}\right)$ versus that of PVDF2 $\left(I_{2}\right)$ on triangular objects of plastic foam and sponge rubber.

material one may feel soft as its thickness is increased, i.e.

$$
S^{F}=l / E
$$

Now, the question is how to make the outputs of PVDF films correspond to the object softness. Three cases were considered and discussed in following.

Case 1: In general the output of PVDF is considered correspondent to the rate of deflection $j \omega \delta_{i}$, that is (referring to Fig. 8),

$$
\begin{aligned}
P V D F 2 & \propto j \omega\left(\alpha_{1} \delta_{1}+\alpha_{2} \delta_{2}\right) \\
& =j \omega u_{1} \frac{\alpha_{1} l_{1} / E_{1}+\alpha_{2} l_{2} / E_{2}}{\sum_{i=1}^{3}\left(l_{i} / E_{i}\right)+l_{4} / E_{4}} \\
& \equiv j \omega u_{1} \frac{a}{c+S_{x}^{F}}
\end{aligned}
$$

$P V D F 1 \propto j \omega\left(\alpha_{2} \delta_{2}+\alpha_{3} \delta_{3}\right)$ 


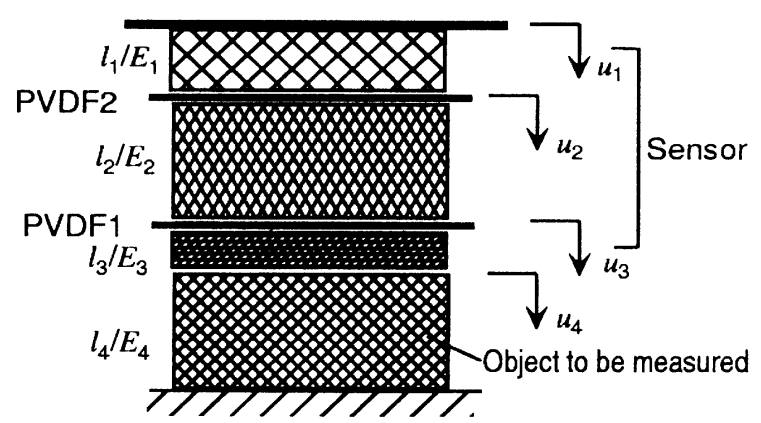

Fig. 8. Analytical model of sensor.

$$
\begin{aligned}
& =j \omega u_{1} \frac{\alpha_{2} l_{2} / E_{2}+\alpha_{3} l_{3} / E_{3}}{\sum_{i=1}^{3}\left(l_{i} / E_{i}\right)+l_{4} / E_{4}} \\
& \equiv j \omega u_{1} \frac{b}{c+S_{x}^{F}}
\end{aligned}
$$

where $\omega$ is the driving frequency of the sensor, $j$ the complex $\sqrt{(-1)}, \alpha_{i}$ coefficients corresponding to the material properties and $S_{x}^{F}=l_{4} / E_{4}$ the softness of the object to be measured.

The above equations show that the outputs of PVDFs are getting higher when the softness $S_{x}^{F}$ goes small and the driving frequency $(\omega)$ or the pushing stroke $\left(u_{1}\right)$ is increased. Figures 3 and 4 are the evidence that the results described in above section can be expressed by eqs.(6) and (7). This, however, is not desirable in practical use because in this case the sensor output is significantly dependent on the pressing stroke and the speed so that the softness could not be assessed if the pressing stroke and speed of the sensor is unknown. Although the effect of the frequency and the pressing stroke can be cancelled by taking a ratio of PVDF2 to PVDF1, coincidentally it leads the softness $S_{x}^{F}$ disappeared from the division of the equation (6) to (7). As an example, if the flat sensor (Fig. 1) was designed being of $l_{1} / E_{1}=l_{3} / E_{3}=l_{2} / 2 E_{2}$, it is easy to get the ratio $\mathrm{PVDF} 2 / \mathrm{PVDF} 1=1$, which is completely independent on the softness of the substance. Figure 5 is positively an experimental proof of the case. So we could say that the equations (6)-(7) are in good agreement with the case of a flat sensor measuring a plane object. Therefore the problem to be solved here is how to make the sensor output independent on the pressing stroke and the speed for practical medical application.

Case 2: If the output of PVDF is made to correspond to the rate of displacement $j \omega u_{i}$, it then gives,

$P V D F 2 \propto j \omega u_{2}$

$$
\begin{aligned}
& =j \omega u_{1} \frac{l_{2} / E_{2}+l_{3} / E_{3}+l_{4} / E_{4}}{\sum_{i=1}^{3}\left(l_{i} / E_{i}\right)+l_{4} / E_{4}} \\
& \equiv j \omega u_{1} \frac{e+S_{x}^{F}}{c+S_{x}^{F}}
\end{aligned}
$$

$$
\begin{aligned}
P V D F 1 & \propto j \omega u_{3} \\
& =j \omega u_{1} \frac{l_{3} / E_{3}+l_{4} / E_{4}}{\sum_{i=1}^{3}\left(l_{i} / E_{i}\right)+l_{4} / E_{4}} \\
& \equiv j \omega u_{1} \frac{f+S_{x}^{F}}{c+S_{x}^{F}}
\end{aligned}
$$

and

$$
\begin{aligned}
\frac{P V D F 2}{P V D F 1} \propto & \frac{e+S_{x}^{F}}{f+S_{x}^{F}} \equiv K_{2} \\
& \Longrightarrow S_{x}^{F}=\frac{e-K_{2} f}{K_{2}-1}
\end{aligned}
$$

It is evident that the softness $S_{x}^{F}$ could be obtained regardless of the pressing stroke and the operating speed. Now the problem comes to how to make the sensor output corresponds to the displacement $u_{i}$ at each PVDF film. Figure 7 has shown the fact that the sensor can distinguish between the sponge rubber and the plastic form if the surface of object is not flat. It gives an important hint that a lump or protuberance on the object may produce a bending deformation on PVDF layer which is being considered relative to the displacement response.

Case 3: In most situations the output of PVDF film can be treated as the combination of Case 1 and Case 2 , that is,

$$
\begin{aligned}
P V D F 2 & \propto j \omega\left(u_{1} \frac{a}{c+S_{x}^{F}}+\beta_{2} u_{1} \frac{e+S_{x}^{F}}{c+S_{x}^{F}}\right) \\
& \equiv j \omega u_{1} \frac{A+\beta_{2} S_{x}^{F}}{c+S_{x}^{F}} \\
P V D F 1 & \propto j \omega\left(u_{1} \frac{b}{c+S_{x}^{F}}+\beta_{1} u_{1} \frac{f+S_{x}^{F}}{c+S_{x}^{F}}\right) \\
& \equiv j \omega u_{1} \frac{B+\beta_{1} S_{x}^{F}}{c+S_{x}^{F}}
\end{aligned}
$$

and

$$
\begin{aligned}
\frac{P V D F 2}{P V D F 1} \propto \frac{\beta_{2}}{\beta_{1}} \frac{\left(A / \beta_{2}\right)+S_{x}^{F}}{\left(B / \beta_{1}\right)+S_{x}^{F}} \equiv K_{3} \\
\Longrightarrow S_{x}^{F}=\frac{K_{3} B-A}{\beta_{2}-K_{3} \beta_{1}}
\end{aligned}
$$



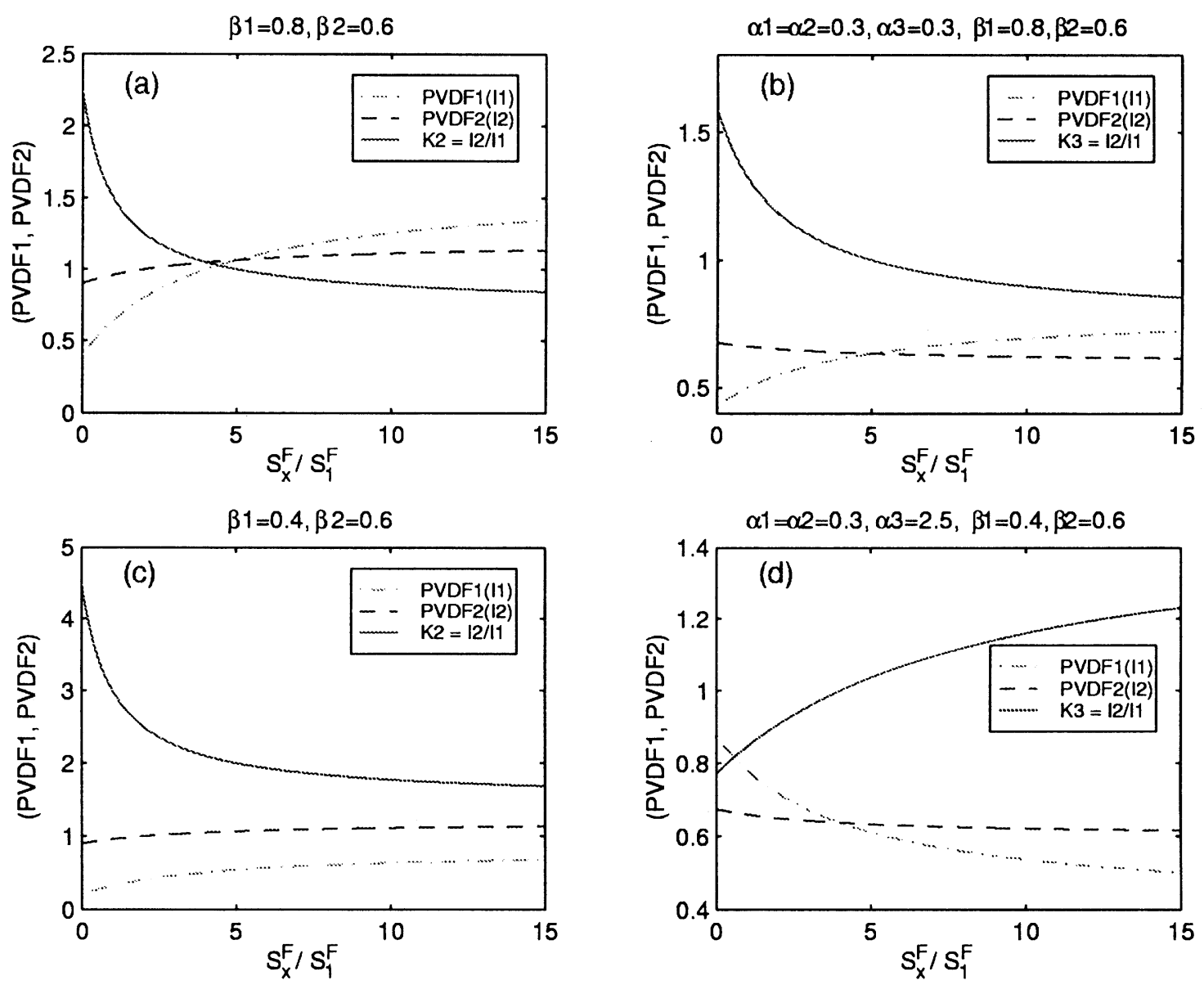

Fig. 9. Simulation of sensor output for Case 2 and Case 3. (a) $\left(\beta_{1}, \beta_{2}\right)=(0.8,0.6)$, (b) $\left(\beta_{1}, \beta_{2}\right)=(0.4,0.6)$, (c) $\alpha_{1}=\alpha_{2}=\alpha_{3}=0.3$ and $\left(\beta_{1}, \beta_{2}\right)=(0.8,0.6),(\mathrm{d}) \alpha_{1}=\alpha_{2}=0.3, \alpha_{3}=2.5$ and $\left(\beta_{1}, \beta_{2}\right)=(0.4,0.6)$.

where $\beta_{i}$ is the coefficient presenting the contribution of the bending deformation to the sensor's output, and it is affected by the softness of the object.

Figure 9 shows the simulation results for Case 2 and Case 3 based on eqs.(10) and (13). The results are obtained by setting $l_{1} / E_{1}=l_{2} / 2 E_{2}=l_{3} / E_{3}$ at a constant and $\alpha_{i}$ and $\beta_{i}$ as variables. The solid lines in the figures display the ratio $K_{2}$ and $K_{3}$. The dashed lines and dashdot lines represent the outputs of PVDF1 and PVDF2 for reference. The lateral axes are the relative softness of the measuring substance to the sensor material $\left(S_{x}^{F} / S_{1}^{F}\right)$. Figures (a) and (c) are the results for Case 2 with $\left(\beta_{1}, \beta_{2}\right)=(0.8,0.6)$ and $(0.4,0.6)$ respectively. Figures.(b) is for Case 3 when the coefficients $\left(\beta_{1}, \beta_{2}\right)=(0.8,0.6)$ and $\alpha_{i}=0.3$ were selected. Comparing with results obtained on a plane sensor against a triangular object (Fig. 7) the simulating resopnse $K_{3}\left(\rightarrow I_{2} / I_{1}\right)$ has the same tendency to the experimental results, in which the $K_{3}$ is decreased gradually when the objects are being soft. Furthermore, Fig. (d) gives an estimation of the output $I_{2} / I_{1}$ when the sensor has the structural parameters by that the coefficients could be, for example, $\alpha_{1}=\alpha_{2}=0.3$, $\alpha_{3}=2.5$ and $\left(\beta_{1}, \beta_{2}\right)=(0.4,0.6)$. In contrast with Fig. (b), the $K_{3}\left(\rightarrow I_{2} / I_{1}\right)$ becomes great gradually with an increases of the softness. This indicates that the sensor structure could change the behavior of its output and the optimal design of the structure should be made individually in order to satisfy different purposes.

Now considering the situation for measuring the softness of prostate gland by rectal palpation, the surface of the intestine is rather smooth and there are no definite tumors or protrusions, hence it is impracticable to palpate the prostate gland across the wall of intestine by a flat surface sensor. According to eq.(13) and the experimental results (Fig. 7), this problem could be solved by placing a protuberance on the sensor, which functions to induce the bending deformation at PVDF film 


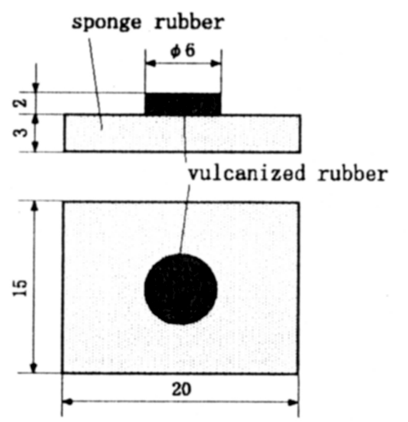

(a) Attachment 1

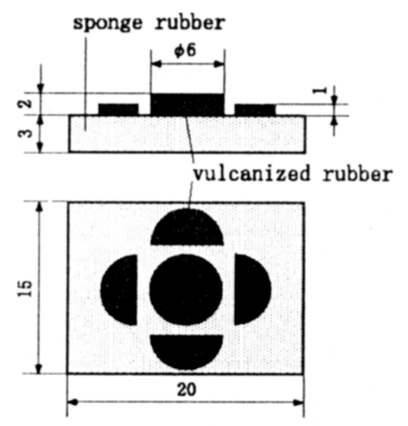

(b) Attachment 2

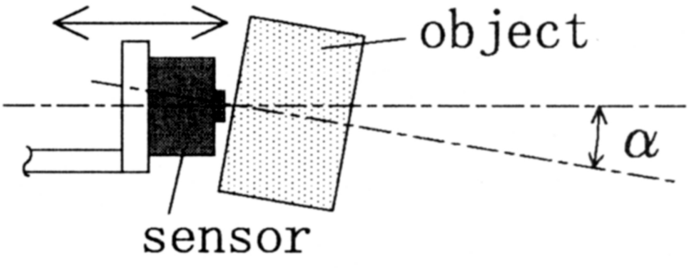

(c) inclination of sensor against object

Fig. 10. Geometry of surface attachments and inclination of sensor against object.

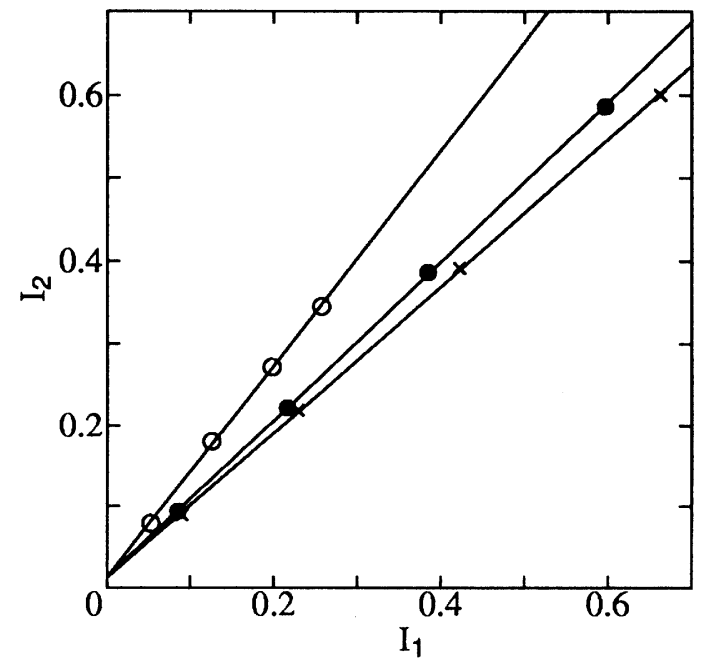

(a) for Attachment 1

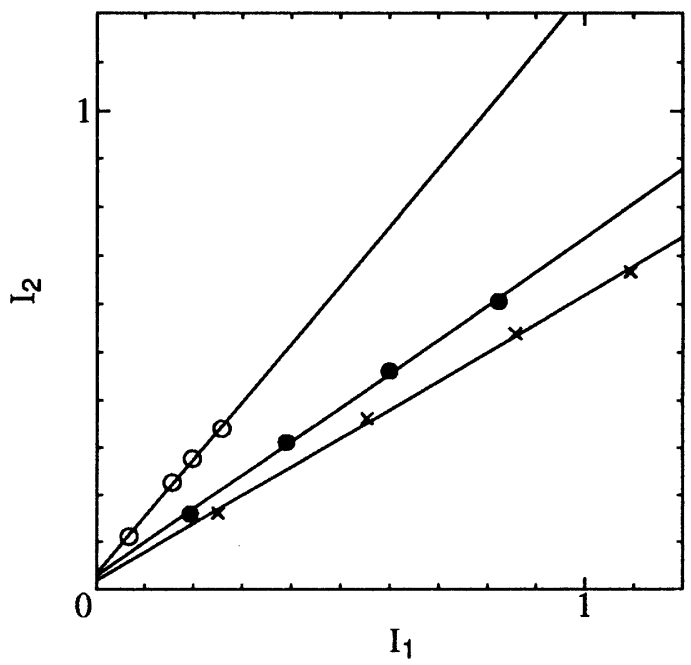

(b) for Attachment 2

Fig. 11. Output of PVDF1 $\left(I_{1}\right)$ versus that of PVDF2 $\left(I_{2}\right)$ for $\alpha=0$ deg. $\bigcirc=$ sponge rubber; $\bigcirc=$ plastic foam; $\times=$ vulcanized rubber.

layers. For detail study, several types of attachments are designed and examined for their sensitivity to distinguish the softness of different substances. Figure 10 illustrates the geometries of two types of attachments, one is a circular vulcanized rubber patch of $\phi 6 \mathrm{~mm}$ in diameter and $2 \mathrm{~mm}$ in thickness stacked on a sponge rubber (Fig. a), and the another is modified by addition of four semi-circular patches of $1 \mathrm{~mm}$ thickness around the circular patch (Fig. b).

Figure 11 illustrates the results obtained as the sensor with the attachments is pressing straightly against three different flat objects: the sponge rubber $(\mathrm{O})$, plastic foam $(\bigcirc)$ and vulcanized rubber $(\times)$. Their regression and correlation coefficients are also calculated and listed in Table 2. Here, the regression coefficient $b$ can be considered corresponding to the coefficient $K_{3}$ of eq.(13). Comparing with Fig. 5, it is clear that adding an attachment on the sensor is an efficient way to improve its sensitivity for assessing the softnesses of flat objects. Figure 12 shows the histories of the output voltages from PVDF1 and PVDF2 as an example when the sensor with Attachment 2 is driven at a constant displacement for measuring the three substances. The output voltages of the PVDF films increase as the measuring substance becomes harder. In detail speaking, the output of PVDF1 increases more significantly than the output of PVDF2 as the hardness of the substance goes up. This implies that the output ratio $I_{2} / I_{1}$ of PVDF2 to PVDF1, see the slope of the lines in Fig. 11, would be in proportion to the softness. Looking at the simulation result in Fig. 9(d), it could say the present sensor with Attachment 1 or 2 has the structural pa- 


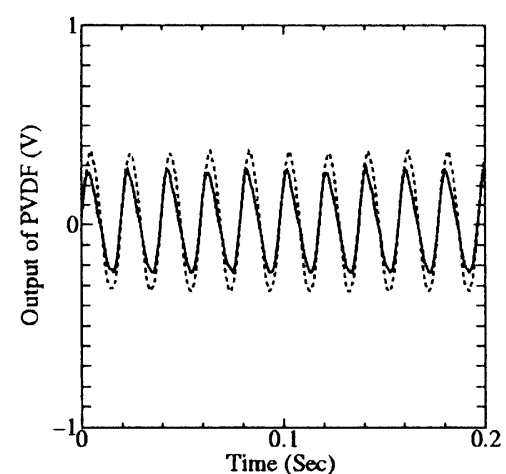

(a) sponge rubber

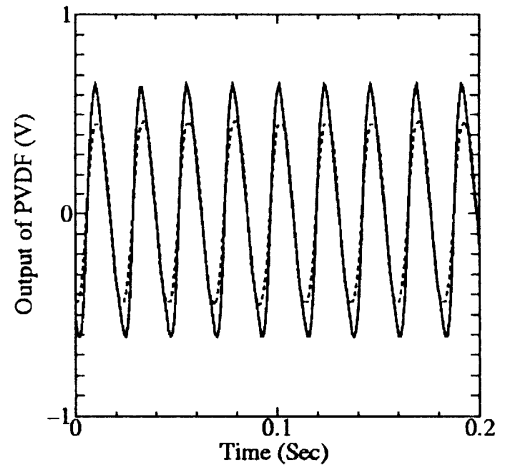

(b) plastic foam

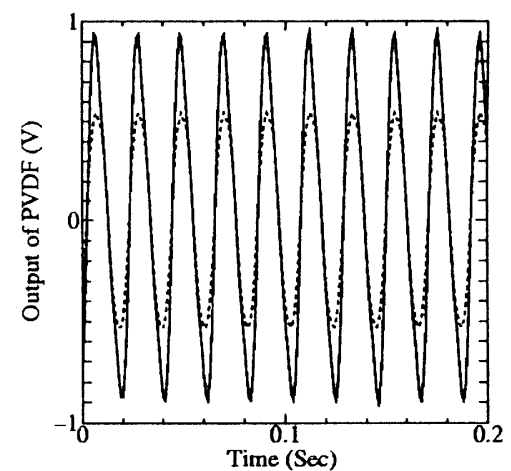

(c) vulcanized rubber

Fig. 12. Voltate output histories of Fig. 11(b). (-) PVDF1, (- - ) PVDF2.

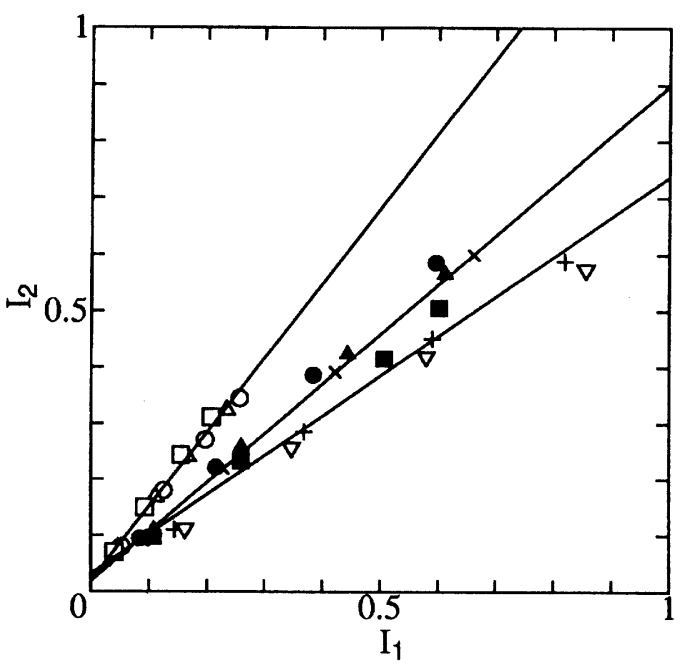

(a) for Attachment 1

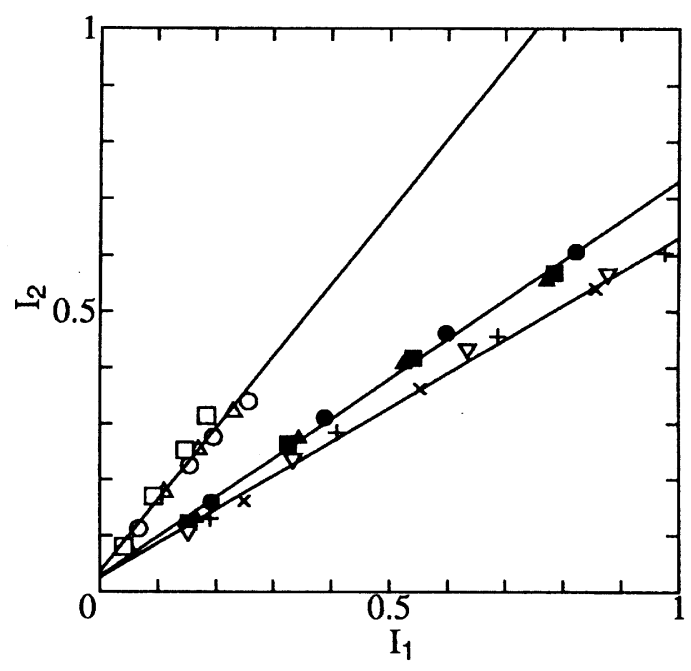

(b) for Attachment 2

Fig. 13. Output of PVDF1 $\left(I_{1}\right)$ versus that of PVDF2 $\left(I_{2}\right)$ as sensor against sponge rubber $(\bigcirc, \Delta, \square)$, plastic foam $(\boldsymbol{O}, \boldsymbol{\Delta}, \boldsymbol{\square})$ and vulcanized rubber $(\times,+, \nabla)$ with inclinations of $\alpha=(0,5,10)$ degrees.

rameters similar to the parameters given in the case of Fig. 9(d). Further, considering eqs.(11-12) and (7) the surface attachments could be mainly described by the structural parameters $\alpha_{3}, \beta_{i}$ and $S_{3}^{F}=l_{3} / E_{3}$. That means one can first change these parameters in analytical model looking for a suitable curve $K_{3}$ which satisfies the substance to be measured, and then designs the attachment based on the simulation results or more detail analysis with add of FEM.

There is anther thing to be verified if the sensor is fitted onto the doctor's forefinger and used inside large intestine. In this case, it is difficult to operate the sensor perpendicular to the wall of the intestine. To investigate the effect on the inclination of the sensor to the
Table 2

Variation of regression coefficients $b$ and correlation coefficients $r$ for $\alpha=0 \mathrm{deg}$

\begin{tabular}{llllll}
\hline & \multicolumn{2}{c}{ Attachment 1 } & & \multicolumn{2}{c}{ Attachment 2 } \\
\cline { 2 - 3 } \cline { 5 - 6 } Specimen & \multicolumn{1}{c}{$b$} & $|r|$ & & $b$ & $|f|$ \\
\hline Sponge rubber & 1.30 & 1.00 & & 1.21 & 0.999 \\
Plastic foam & 0.962 & 1.00 & & 0.708 & 0.999 \\
Vulcanized rubber & 0.890 & 1.00 & & 0.600 & 0.999 \\
\hline
\end{tabular}

object, additional experiments were carried out considering the inclination $\alpha$ to be fluctuated within $10 \mathrm{de}-$ grees. The obtained results are shown in Fig. 13 and the corresponding regression $b$ and correlation $r$ coefficients are in Table 3. It is clear that the three materials can be distinguished from each other by the ratio 


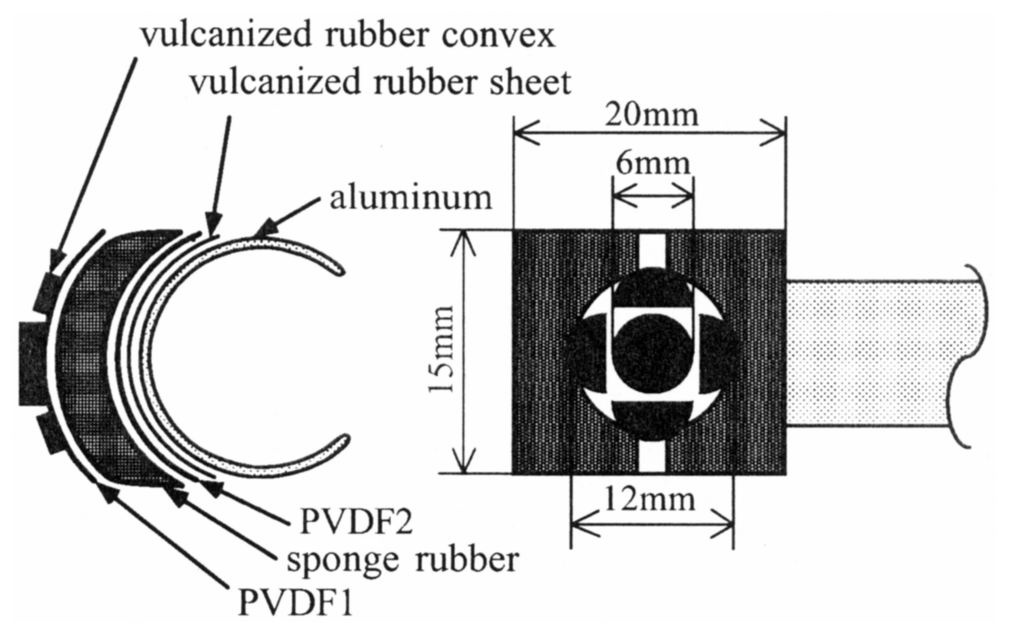

Fig. 14. Cylindrical sensor with Attachment 2 designed for fitting on forefinger.

Table 3

Variation of regression coefficients $b$ and correlation coefficients $r$ for $\alpha=0,5,10 \mathrm{deg}$

\begin{tabular}{lcccccc}
\hline & \multicolumn{2}{c}{ Attachment 1 } & & \multicolumn{2}{c}{ Attachment 2 } \\
\cline { 2 - 3 } \cline { 5 - 6 } Specimen & $b$ & $|r|$ & & $b$ & $|f|$ \\
\hline Sponge rubber & 1.32 & 0.994 & & 1.28 & 0.978 \\
Plastic foam & 0.880 & 0.989 & & 0.703 & 0.998 \\
Vulcanized rubber & 0.706 & 0.972 & & 0.605 & 0.998 \\
\hline
\end{tabular}

$I_{2} / I_{1}$ or the regression coefficient $b$ almost regardless of the inclination in operating. Further comparing the correlation coefficients $r$ in Table 3 on Attachment 1 and 2, it may come to a conclusion that Attachment 2 gives a output more sensitive and reliable. Hence, the structure of Attachment 2 is adopted for design of a prostatic palpation probe in following section.

\section{Prostatic palpation probe and clinical test}

A probe for prostatic palpation fabricated is shown in Fig. 14. The sensor consists of an aluminum open cylindrical shell, thin vulcanized rubber of $1 \mathrm{~mm}$ thickness, PVDF film, sponge rubber layer of $3 \mathrm{~mm}$ thickness, the second layer of PVDF film and Attachment 2, which are piled up in sequence.

In the clinical test the doctor first examined the softness of the prostate gland using his own forefinger, and then the same rectal palpation was made by the sensor which was installed onto the forefinger. The signals from the sensor were recorded to a computer, and they are calculated for quantification of the forefinger palpation results.

Figure 15 presents the results obtained from clinical test to more than 30 patients. The symbol in

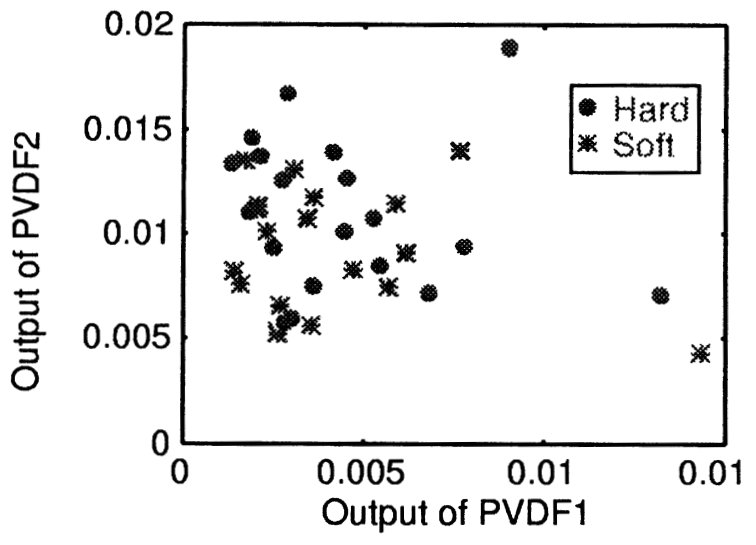

Fig. 15. Plot of indexes $I_{1}$ and $I_{2}$ calculated by eq.(1) (clinical test in rectal palpation).

the figure indicates the patients whose prostate gland was diagnosed 'hard' or cancer, and the symbol $*$ for those whose prostates were found out 'soft' or normal. The indexes $I_{1}$ and $I_{2}$ were calculated by eq.(1) directly from the original measuring data. It is obvious that the distinction between 'hard' and 'soft' cannot be made from the graph. However, the sensor has shown its efficiency for assessment of hardness or softness of substances in above section. What happened in rectal palpation with the sensor can be interpreted in various ways, for examples, the difficulty to specify the same position in palpation by the forefinger and the senor, the different shape and size of the glands for each individual person, and so on. The major reason may be considered that it is not easy to keep the sensor in a constant harmonic motion by the forefinger in rectum.

As a proof, Fig. 16 shows a pair of sample signals obtained from the sensor in rectal palpation. The first 

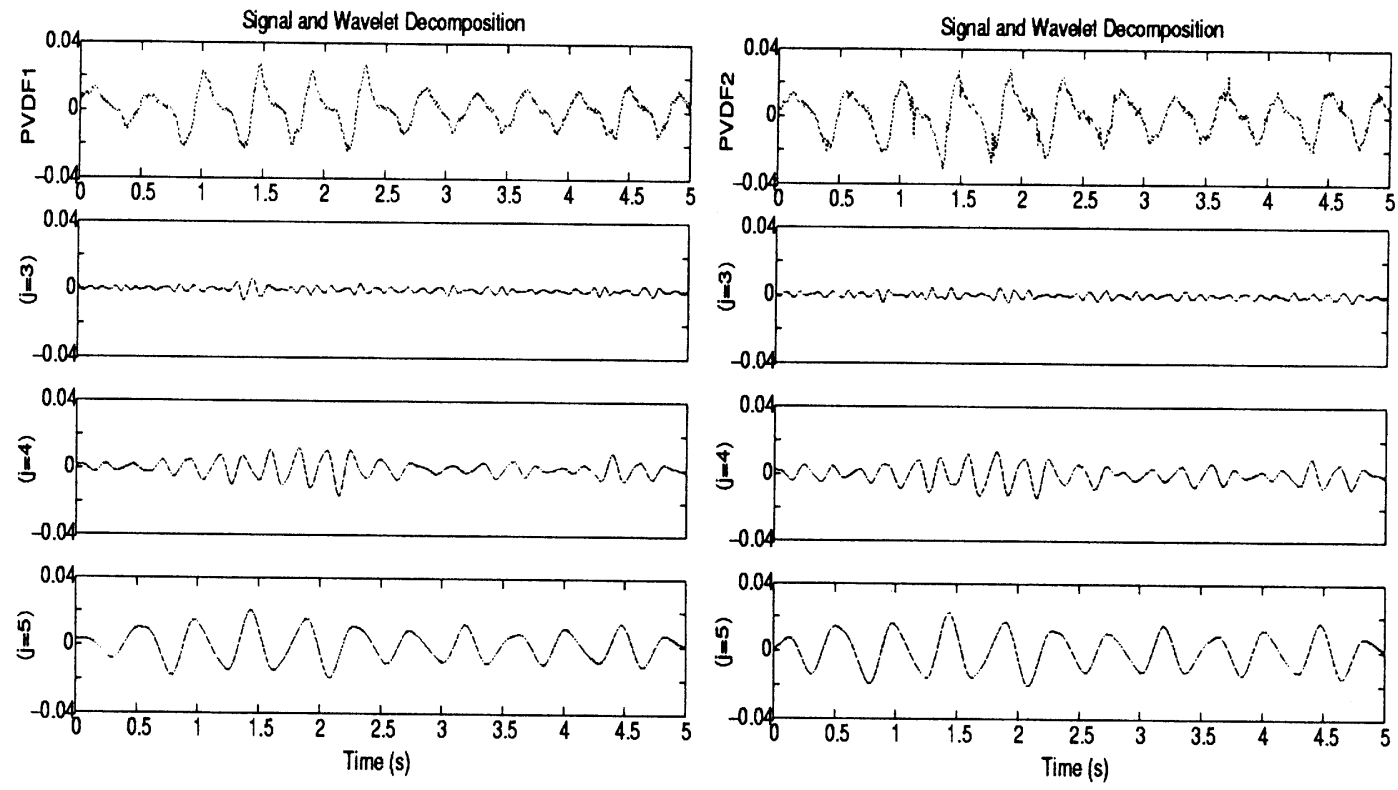

Fig. 16. An example of wavelet decompositions on prostate palpation signals.

columns in the figure are the time histories of the palpation signal. Comparing with the signals obtained, for example, from a sponge rubber block (Fig. 12), the signals of rectal palpation involved a big noise, especially some high frequency components. That means the sensor did not operated in a constant harmonic motion. Therefore, it is evident that the indexes $I_{k}$ will lose its accuracy for evaluation of the softness if the original signal is calculated directly by eq.(1).

In order to extract the contributed component from the real signals, the discrete wavelet decomposition method was introduced to deal with the signals. Suppose $f(i)$ is a series of discrete signal obtained from the sensor, it is expressed by the wavelet transform as,

$$
f(i) \sim \sum_{j} \sum_{k} d_{k}^{(j)} \psi\left(2^{j} i-k\right)
$$

Here, $d_{k}^{(j)}$ is the wavelet coefficient and $\psi$ is the Symlets wavelet 'Sym8' [1] which is selected as the mother wavelet in following analysis. The wavelet decomposition at level $j$ is then calculated by

$$
g_{j}(i)=\sum_{k} d_{k}^{(j)} \psi\left(2^{j} i-k\right)
$$

Further, the index $I_{n}$ at level $j$ is defined by

$$
I_{n}=\sum_{i}\left|g_{j}(i)\right|, \mathrm{n}=1,2 \text { for PVDF1, PVDF2 (16) }
$$

Three wavelet decompositions at level $j=3$ to 5 were plotted at the next columns in Fig. 16. The central frequency at level $j=5$ is about $3 \mathrm{~Hz}$ and $6 \mathrm{~Hz}$ at level $j=4,12 \mathrm{~Hz}$ at level $j=3$. Since the decomposed signals at level $j=5$ are obviously corresponding to the sensor's operating movement, the index $I_{n}$ are calculated from the decompositions at level $j=5$ and are plotted in Fig. 17. As contrasted with Fig. 15, it is obvious that the indexes $I_{1}$ and $I_{2}$ are concentrated at the small value region for the soft prostate glands. This means we can set a border, for instance, the broken line to distinguish the normal or abnormal glands. In this case, 11 soft glands are lain within the soft region, by which the patients are declared to be normal. However, the others whose glands are lain on or outside the border could be considered abnormal and a close examination would be requested. Although the results are not as much good as expected and the advanced development of the sensor is required, if the soft region border line has been determined suitably based on the safety coefficient, the present palpation sensor could be used for group health examination on the prostate gland to give a preliminary differentiation of the normal prostate glands from the prostatic cancers.

\section{Conclusions}

A theoretical and experimental study on the prostatic palpation sensor has been presented. The sensor was made in a layered medium with two polyvinylidene 


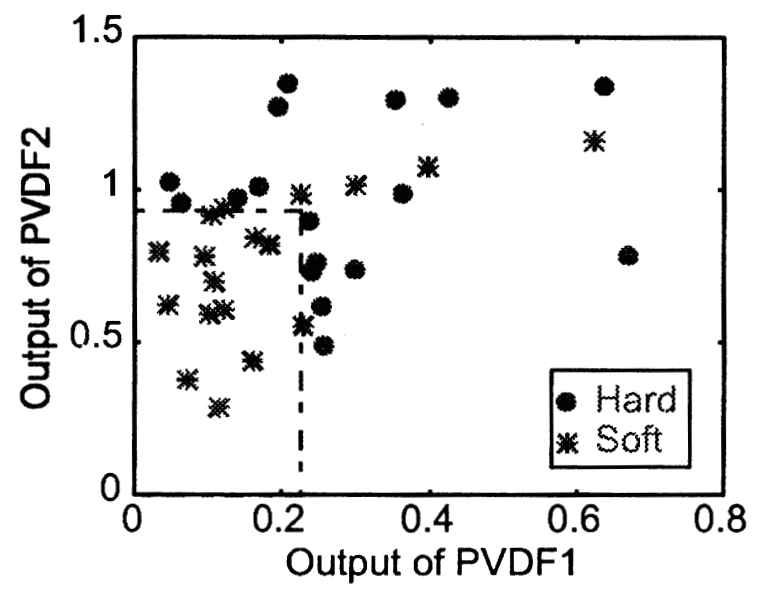

Fig. 17. Plot of indexes $I_{1}$ and $I_{2}$ calculated by eq.(16) (clinical test in rectal palpation).

fluoride (PVDF) films and the ratio of the two PVDF outputs was validated for assessment of the substance softness if a suitable attachment is designed. The theoretical qualitative analysis on the sensor structure was introduced and the theoretical sensor model could be useful for the sensor structure design. It was also denoted that the softness defined by the thickness and Young's Modulus of the substance is more reasonable to express human's touch sense.

Further, a compact cylindrical sensor was fabricated and the rectal palpation of the prostate gland could be carried out by fitting the sensor onto the doctor's forefinger so that digital signals can be measured and recorded for detail analysis. The sensor proposed here is verified, by the preliminary clinic test on more than 30 patients, to be a useful tool for group examination on prostate glands.

\section{Acknowledgement}

The authors gratefully acknowledge that a part of this work was subsidized by Izumi Science and Technology Foundation and Toyota Physical \& Chemical Research Institute, and authors also thank AMP Technology Japan Ltd. for their supply of PVDF film samples.

\section{References}

[1] J. Buckheit, S. Chen, D. Donoho, I. Johnstone and J. Scargle, About WaveLab, Stanford University, http://playfair.stanford.edu/wavelab. 1995, Version 0.700

[2] M. Gniadecka and J. Serup, Suction Chamber Method for
Measurement of Skin Mechanical Properties: The Dermaflex, CRC Press, 1995, pp. 329-334.

[3] Z.W. Jiang, S. Chonan, Y. Tanahashi and Y. Suga, Tactile Sensor for Detection of Prostatic Hypertrophy, Journal of the Japan Society of Applied Electromagnetics and Mechanics 4(3) (1996), 23-28.

[4] I. Kato, K. Koganezawa and A. Takanishi, Automatic Palpation System for Breast Cancer: WAPRO-4, Journal of the Robotics Society of Japan 5(2) (1987), 102-108.

[5] T. Kosawada, R. Hamaguchi, Y. Suzuki, Y. Kubota, T. Nakada, K. Suzuki and G. Shikanai, A Method to Detect Dynamic Characterisitics of Small Biological Soft Tissues and Its Application to Human Prostate Tissue, Transactions of the Japan Society of Mechanical Engineers, Series C 61(591) (1995), 4410-4416.

[6] S. Omata, Development of Piezoelectric Transducer for Measuring Contact Compliance of a Soft Body, Japanese Journal of Medical Electronics and Biological Engineering 24(5) (1986), 330-334.

[7] S. Omata, Characteristics of the Vibration Modes and the Contact Compiances of a Soft Material Measured with a Newly Developed Piezoelectric Vibrometer, Japanese Journal of Medical Electronics and Biological Engineering 26(1) (1988), 3240 .

[8] S. Omata, Measurements of the Hardness of a Soft Material with a Piezoelectric Vibrometer and Their Analysis, Japanese Journal of Medical Electronics and Biological Engineering 28(1) (1990), 1-8.

[9] J. Rigal and J.L. Leveque, In vivo measurement of the Stratum Corneum Elasticity, Bioengineering and the Skin 1 (1985), 13-23.

[10] G.M. Shepherd, Neurobiology (Third Edition), Oxford University Press, 1994

[11] H. Tanaka, H. Ujiie, R. Aoyagi, N. Hoshimiya and Y. Handa, Basic Study of the Muscle Stiffness Measurement by Ultrasonic Vibrator, Japanese Journal of Medical Electronics and Biological Engineering 32(1) (1994), 1-7.

[12] K. Yoshida and T. Hayashi, Study on Flexible Hardness Sensor for Cancer Palpation in Human Body: Proposition of Balloontype Sensor and its Experiments, Journal of the Japan Society for Precision Engineering 58(11) (1992), 105-110. 

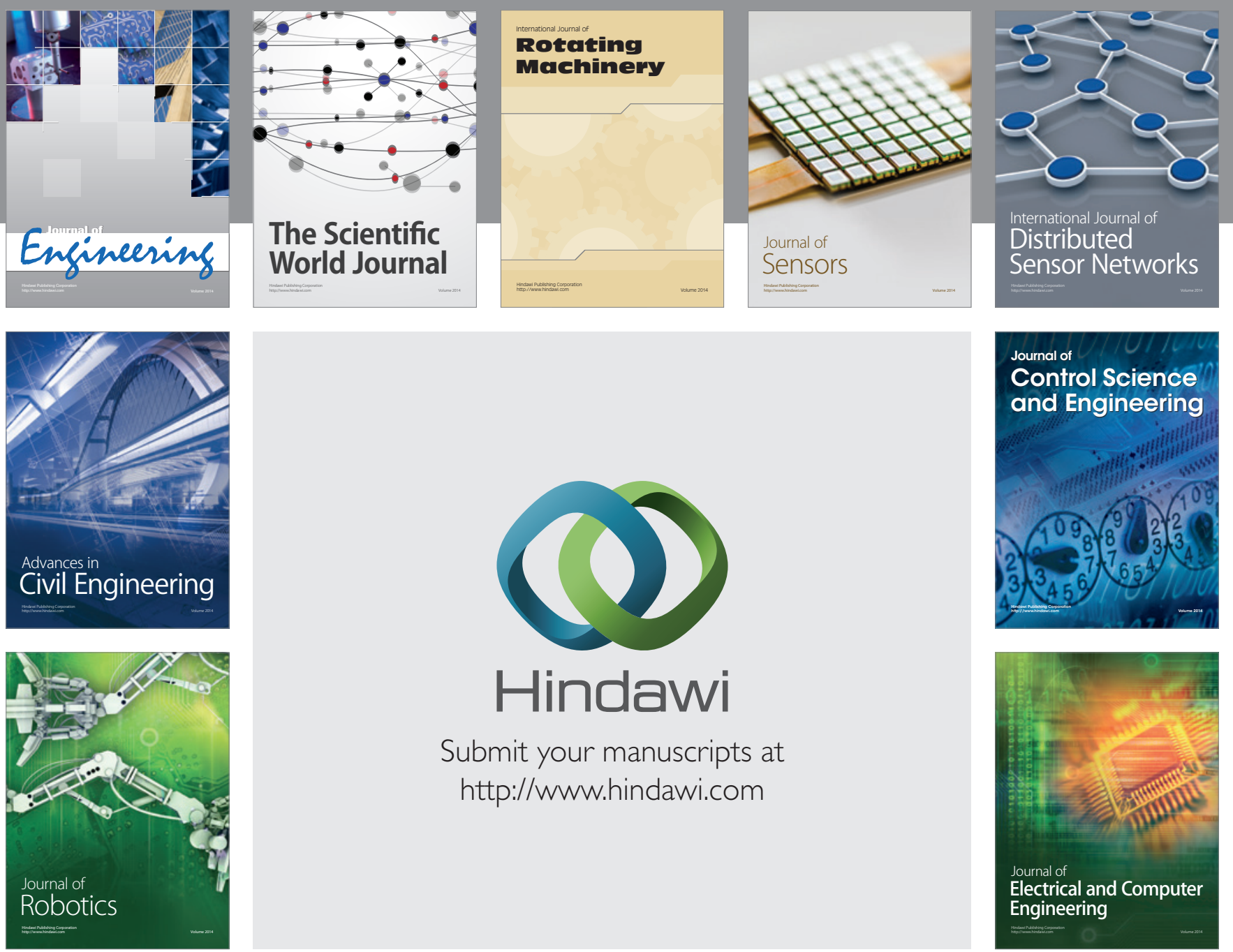

Submit your manuscripts at

http://www.hindawi.com
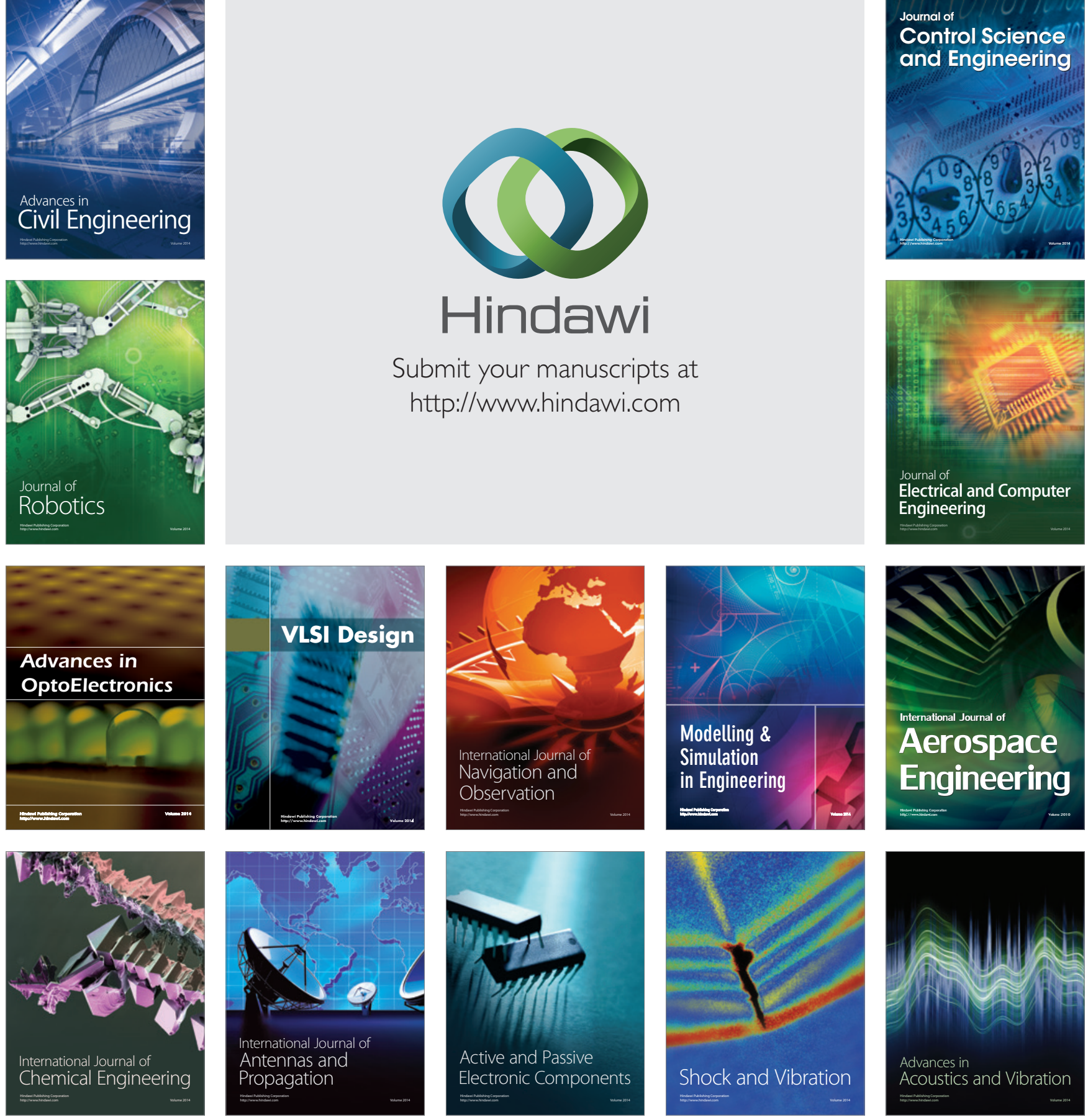